\section{Twelve women from the National Register of Antipsychotic Medication in Pregnancy}

\section{K McCauley-Elsom, J Kulkarni}

Alfred Psychiatry Research Centre, The Alfred and Monash University, School of Psychology, Psychiatry \& Psychological Medicine, Melbourne, Australia

Background: The National Register of Antipsychotic Medication in Pregnancy (NRAMP) has been established in Australia. The use of antipsychotic medications in pregnancy remains a dilemma for clinicians who must weigh up the risk of harm to the developing baby against the risk of relapse for the mother if medications are altered or ceased. The literature continues to present the difficulty experienced in maintaining the well-being of women with a history of serious mental illness during the perinatal period. The establishment of the NRAMP has required the collaboration of a variety of health care professional around the country both to attain the multicentered ethical approval and to recruit participants.

Methods: A case study approach will present a description of the first 13 women on the NRAMP.

Results: Using the case study method, the information presented will include pregnancy pathways, birth outcomes, postnatal outcomes and episodes of separation of mother and baby. An outline of medication use in pregnancy along with the pregnancy outcomes, to date, will be presented.

Conclusions: This presentation introduces the first outcomes from the NRAMP, using a case study methodology. It clearly identifies the complexity of the issues surrounding the management of women who have a history of psychosis during the perinatal period.

\section{Prevalence and course of depression in cognitively intact and cognitively impaired nursing home residents}

\section{K McSweeney, D O'Connor}

Monash University, Melbourne, Australia

Background: This research investigated the prevalence and course of depression in newly admitted nursing home residents. Representing a departure from the majority of prior research, consecutive admissions were recruited into the study, irrespective of cognitive status. This enabled a comparison of the prevalence and course of depression experienced by cognitively intact residents and those exhibiting all levels of cognitive impairment.
Methods: Depression was assessed at 1, 3 and 6 months postadmission. The assessment entailed the conduct of a semistructured clinical interview, which allowed for the provision of DSM-IV diagnoses and the scoring of the Cornell Scale for Depression in Dementia.

Results: Participants were 51 newly admitted residents, drawn from six nursing homes. At admission, nearly $25 \%$ of residents were diagnosed with major depression (MD), and a further $20 \%$ evidenced a nonmajor depressive disorder. At the second and third assessments, MD was observed in $14 \%$ and $15 \%$ of residents, respectively. Of particular interest, only the cognitively impaired were diagnosed with MD during the study. For residents who completed all three assessments, there was no appreciable change in the levels of depression apparent.

Conclusions: The current study showed that a great many nursing home residents experience severe depressions that are unlikely to remit spontaneously. Most often, these residents exhibited pronounced cognitive impairment. Accordingly, care staff and GPs must be trained in the identification of depression in dementia, and any interventions implemented in these facilities should be tailored to meet the unique needs of this group.

\section{Mental health literacy in a community sample of women with eating disorders}

\author{
J Mond', C Owen'², B Rodgers'², P Hay' \\ 'James Cook University, Townsville, Australia; and ${ }^{2}$ Australian National University, \\ Canberra, Australia
}

Background: Individuals with bulimia nervosa (BN) and related disorders typically do not receive treatment for an eating problem. Poor mental health literacy may account for this. We examined mental health literacy relating to $\mathrm{BN}$ in a community sample of young adult women $(n=158)$ with bulimic-type eating disorders.

Methods: A vignette was presented describing a fictional person meeting diagnostic criteria for BN. A series of questions followed in which participants were asked about the nature and treatment of the problem described.

Results: Primary care practitioners, psychologists, counselors and close friends were perceived as helpful in the treatment of the problem described by the vast majority of participants. Self-help interventions, including taking vitamins and minerals, also were highly regarded, whereas participants were ambivalent about the benefits of psychiatrists and antipathetic toward the use of prescription medication. Most participants believed that the problem would be difficult to treat, 